\title{
Novel and unexpected cellular partners for HIV-I integrase and Tat
} Mauro Giacca

\author{
Address: International Centre for Genetic Engineering and Biotechnology (ICGEB), Trieste, Italy \\ from Frontiers of Retrovirology: Complex retroviruses, retroelements and their hosts \\ Montpellier, France. 2I-23 September 2009 \\ Published: 24 September 2009 \\ Retrovirology 2009, 6(Suppl 2):18 doi:10.1 186/1742-4690-6-S2-18
}

This abstract is available from: http://www.retrovirology.com/content/6/S2/l8

(c) 2009 Giacca; licensee BioMed Central Ltd.

Over the last few years, we have been interested in the identification of the relevant cellular partners mediating function of HIV-1 Tat and integrase.

Long standing evidence indicates that quiescent human peripheral blood CD4+ T-cells do not support efficient HIV replication, since viral infection halts at a step that precedes integration of the HIV-1 cDNA into the host genome. We discovered that permissivity of activated cells to HIV-1 infection is regulated by a novel, sequential posttranslational pathway of protein modification that regulates viral DNA integration. We found that, in activated T lymphocytes, viral integrase (IN) is phosphorylated by the cellular c-Jun N-terminal Kinase (JNK) on a highly conserved serine residue in its core domain. Phosphorylated IN in turn becomes a substrate for the cellular peptidyl prolylisomerase enzyme Pin-1, which catalyzes a conformational modification of IN. These concerted activities increase integrase stability and are a requisite for efficient HIV-1 integration and infection. Lack of these modifications in resting CD4+ T-cells due the absence of JNK function essentially restricts viral infection in these cells.

Parallel work has aimed at assessing the molecular interactions mediating HIV-1 Tat function. We have long been intrigued by the peculiar property of the protein to be released by the cells through a non-canonical, ER-Golgiindependent protein secretion pathway and to enter neighboring cells, where it is transported to the nucleus in a transcriptionally active form. Of note, fusion of heterologus proteins to the Tat basic domain also imparts these proteins the intercellular trafficking capacity of the wild type protein. By assessing the effect of a series of metabolic inhibitors on leaderless Tat release, we have now mapped the relevant cellular interactions mediating extracellular Tat release. 